\title{
System Study:
}

High-Pressure Coolant Injection

1998-2018

Zhegang Ma

Kellie J. Kvarfordt

John A. Schroeder

Thomas E. Wierman

December 2019

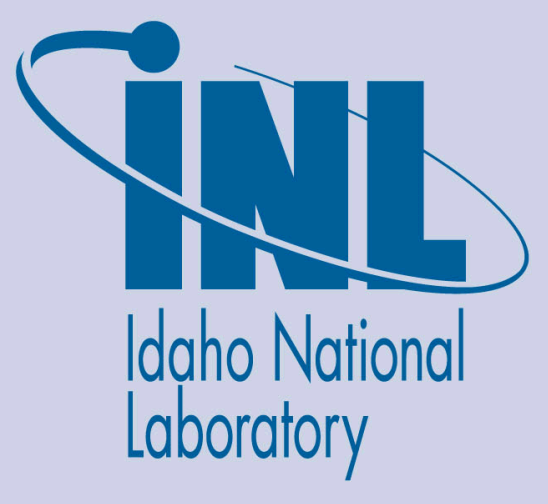

The INL is a U.S. Department of Energy National Laboratory operated by Battelle Energy Alliance 
NOTICE

This information was prepared as an account of work sponsored by an agency of the U.S. Government. Neither the U.S. Government nor any agency thereof, nor any of their employees, makes any warranty, express or implied, or assumes any legal liability or responsibility for any third party's use, or the results of such use, of any information, apparatus, product, or process disclosed herein, or represents that its use by such third party would not infringe privately owned rights. The views expressed herein are not necessarily those of the U.S. Nuclear Regulatory Commission. 


\title{
System Study: High-Pressure Coolant Injection 1998-2018
}

\author{
Zhegang Ma ${ }^{1}$, Kellie J. Kvarfordt ${ }^{1}$, \\ John A. Schroeder', Thomas E. Wierman ${ }^{2}$
}

Update Completed December 2019

\begin{abstract}
${ }^{1}$ Idaho National Laboratory, Risk Assessment and Management Services Department, Idaho Falls, Idaho 83415

${ }^{2}$ Schroeder Incorporated, 3945 Wise Owl Way, Island Park, Idaho 83429
\end{abstract}

http://www.inl.gov

Prepared for the Division of Risk Assessment Office of Nuclear Regulatory Research

U.S. Nuclear Regulatory Commission NRC Agreement Number NRC-HQ-14-D-0018 



\begin{abstract}
This report presents an unreliability evaluation of the high-pressure coolant injection system (HPCI) at 25 U.S. commercial boiling water reactors. Demand, run hours, and failure data from 1998 through 2018 for selected components were obtained from the Institute of Nuclear Power Operations (INPO) Industry Reporting and Information System (IRIS), formerly the INPO Consolidated Events Database (ICES). The unreliability results are trended for the most recent 10 -year period while yearly estimates for system unreliability are provided for the entire active period. No statistically significant increasing or decreasing trends were identified in the HPCI results.
\end{abstract}




\section{CONTENTS}

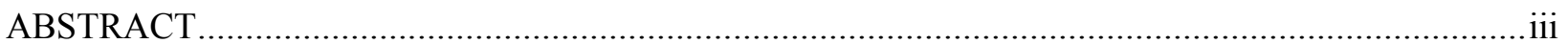

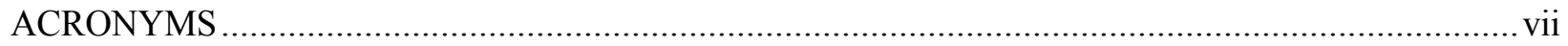

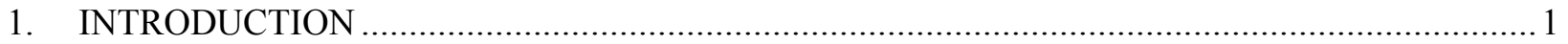

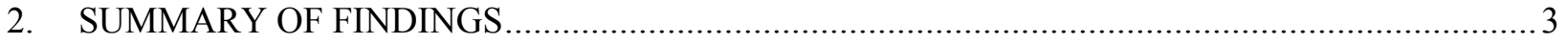

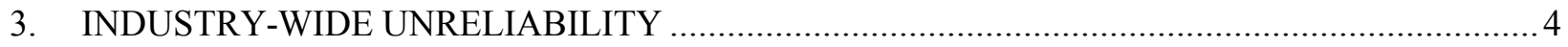

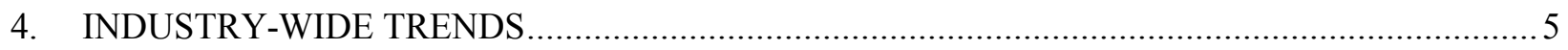

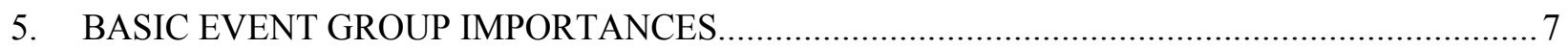

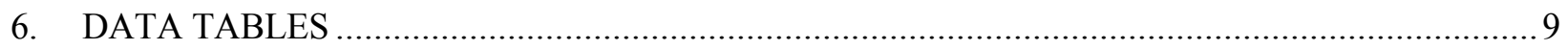

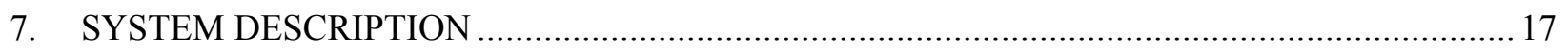

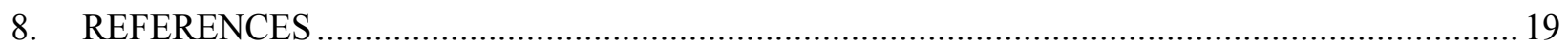

\section{FIGURES}

Figure 1. Trend of HPCI system unreliability (start-only model), as a function of fiscal year................. 6

Figure 2. Trend of HPCI system unreliability (8-hour model), as a function of fiscal year. ................... 6

Figure 3. HPCI basic event group importances...................................................................... 7

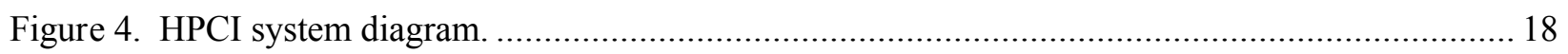

TABLES

Table 1. BWR plants with a HPCI system selected for the study............................................... 2

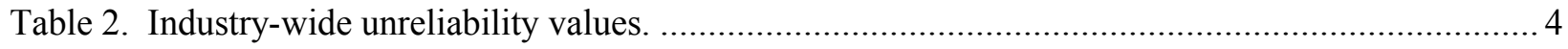

Table 3. HPCI model basic event importance group descriptions................................................... 8

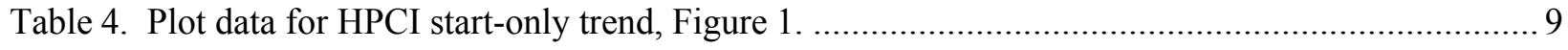

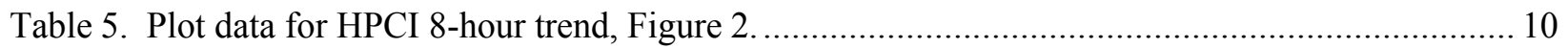




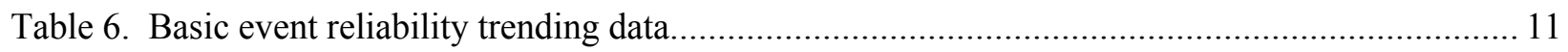

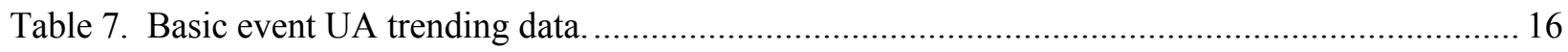

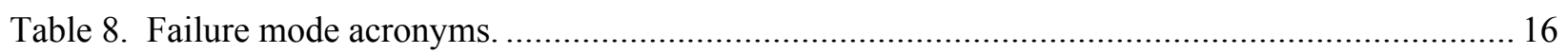




\title{
ACRONYMS
}

\author{
BWR boiling water reactor \\ CCF common-cause failure \\ CST condensate storage tank \\ EPIX Equipment Performance and Information Exchange \\ FTOC fail to open/close \\ FTOP fail to operate \\ FTR $>1 \mathrm{H}$ fail to run more than one hour (standby) \\ $\mathrm{FTR}<1 \mathrm{H}$ fail to run less than one hour (after start) \\ FTS fail to start \\ HPCI high-pressure coolant injection \\ ICES INPO Consolidated Events Database \\ INPO Institute of Nuclear Power Operations \\ IRIS Industry Reporting and Information System \\ MOV motor-operated valve \\ MSPI Mitigating Systems Performance Index \\ NRC Nuclear Regulatory Commission \\ PRA probabilistic risk assessment \\ ROP Reactor Oversight Process \\ RPV reactor pressure vessel \\ SPAR standardized plant analysis risk \\ SSU safety system unavailability
}

UA unavailability (maintenance or state of another component) 
System Study

High-Pressure Coolant Injection viii

2018 Update

December 2019 


\section{System Study: High-Pressure Coolant Injection 1998-2018}

\section{INTRODUCTION}

This report presents an unreliability evaluation of the high-pressure coolant injection (HPCI) systems at the 25 U.S. commercial boiling water reactors (BWRs) listed in Table 1. For each plant, the corresponding Standardized Plant Analysis Risk (SPAR) model (version model indicated in Table 1) was used in the yearly calculations. Demand, run hours, and failure data from 1998 through 2018 for selected components in the HPCI system were obtained from the Institute of Nuclear Power Operations (INPO) Industry Reporting and Information System (IRIS), formerly the INPO Consolidated Events Database (ICES) and the Equipment Performance and Information Exchange Database (EPIX). Train unavailability data (outages from test or maintenance) were obtained from the Reactor Oversight Process (ROP) Safety System Unavailability (SSU) database (1998 through 2001) and the Mitigating Systems Performance Index (MSPI) database (2002 through 2018). Common-cause failure (CCF) data used in the models are from the 2010 update to the CCF database. The system unreliability results are trended for the most recent 10-year period while yearly estimates for system unreliability are provided for the entire active period.

This report does not attempt to estimate basic event values for use in a probabilistic risk assessment (PRA). Suggested values for such use are presented in the 2015 Component Reliability Update [1], which is an update to NUREG/CR-6928 [2] and the 2010 Component Reliability Update [3]. Baseline HPCI unreliability results using basic event values from the 2010 Component Reliability Update ${ }^{a}$ are summarized in Section 3. Trend results for HPCI (using system-specific data) are presented in Section 4. Similar to previous system study updates, Section 5 contains importance information (using the baseline results from Section 3), and Section 7 describes the HPCI.

The HPCI model is evaluated using the transient flag set in the SPAR model. The transient flag set assumes all support systems are available and that the HPCI system is required to perform to mitigate the effects of the transient initiating event. All models include failures due to unavailability while in test or maintenance. Human error and recovery events in the models are set to False in the study for the results to represent the mechanical part of the system has not been included in the SPAR model logic. An overview of the trending methods, glossary of terms, and abbreviations can be found in the paper Overview and Reference [4] on the Nuclear Regulatory Commission (NRC) Reactor Operational Experience Results and Databases web page (https://nrcoe.inl.gov/resultsdb/).

Two modes of the models for the HPCI system are calculated. The HPCI start-only model is the HPCI SPAR model modified by setting all fail-to-run basic events to zero (False), setting all human error and recovery events to False, setting the suction transfer to the torus to False, and setting all cooling basic events to False. The 8-hour mission model sets all human error and recovery events to False.

\footnotetext{
${ }^{\text {a }}$ In order to keep the SPAR models and BE data static so that the only variable in the analysis was the demand, run hours, failure, and unavailability data for selected components in the HPCI system, the same versions of SPAR models as those in the 2016 system study were used in this study. Those versions of SPAR models used data from the 2010 Component Reliability Update.
} 
Table 1. BWR plants with a HPCI system selected for the study.

\begin{tabular}{lc}
\hline \multicolumn{1}{c}{ Plant } & Version \\
\hline Browns Ferry 1 & 8.22 \\
\hline Browns Ferry 2 & 8.22 \\
\hline Browns Ferry 3 & 8.18 \\
\hline Brunswick 1 & 8.20 \\
\hline Brunswick 2 & 8.20 \\
\hline Cooper & 8.22 \\
\hline Dresden 2 & 8.18 \\
\hline Dresden 3 & 8.18 \\
\hline Duane Arnold & 8.22 \\
\hline Fermi 2 & 8.20 \\
\hline FitzPatrick & 8.17 \\
\hline Hatch 1 & 8.20 \\
\hline Hatch 2 & 8.20 \\
\hline Hope Creek & 8.18 \\
\hline Limerick 1 & 8.20 \\
\hline Limerick 2 & 8.19 \\
\hline Monticello & 8.20 \\
\hline Peach Bottom 2 & 8.25 \\
\hline Peach Bottom 3 & 8.21 \\
\hline Pilgrim & 8.21 \\
\hline Quad Cities 1 & 8.18 \\
\hline Quad Cities 2 & 8.18 \\
\hline Susquehanna 1 & 8.23 \\
\hline Susquehanna 2 & 8.21 \\
\hline Vermont Yankee & 8.19 \\
\hline
\end{tabular}




\section{SUMMARY OF FINDINGS}

The results of this HPCI system unreliability study are summarized in this section. Of particular interest is the existence of any statistically significant ${ }^{\mathrm{a}}$ increasing trends. In this update, no statistically significant increasing or decreasing trends were identified in the HPCI unreliability trend results.

The industry-wide HPCI start-only and 8-hour basic event group importances were evaluated and are shown in Figure 3:

- In the Start-Only case - the leading contributor to HPCI system unreliability is the HPCI TDP group of basic events followed by the Injection group.

- In the 8-Hour case - the leading contributor to HPCI system unreliability is also the HPCI TDP group of basic events followed by the Injection group.

a. Statistically significant is defined in terms of the 'p-value.' A p-value is a probability indicating whether to accept or reject the null hypothesis that there is no trend in the data. P-values of less than or equal to 0.05 indicate that we are $95 \%$ confident that there is a trend in the data (reject the null hypothesis of no trend.) By convention, we use the "Michelin Guide" scale: p-value $<0.05$ (statistically significant), p-value $<0.01$ (highly statistically significant); pvalue $<0.001$ (extremely statistically significant). 


\section{INDUSTRY-WIDE UNRELIABILITY}

The HPCI fault trees from the SPAR models were evaluated for each of the 25 operating U.S. commercial boiling water nuclear power plants with a HPCI system.

The industry-wide unreliability of the HPCI system has been estimated for two modes of operation. A failure to start (start-only) model and an 8-hour mission model were evaluated. The uncertainty distributions for HPCI show both plant design variability and parameter uncertainty from the industrywide component failure data (1998-2010). ${ }^{\mathrm{a}}$

Table 2 shows the percentiles and mean of the aggregated sample data (Latin hypercube, 1000 samples for each model) collected from the uncertainty calculations of the HPCI fault trees in the SPAR models. The lower and upper bounds are based directly on the samples (Latin hypercube) from the uncertainty calculations in the SPAR models. For the industry-level results, the SPAR samples were combined into one large sample in order to determine the industry-level bounds, mean, and median.

Table 2. Industry-wide unreliability values.

\begin{tabular}{ccccc}
\hline Model & Lower (5\%) & Median & Mean & Upper (95\%) \\
\hline Start-Only & $1.49 \mathrm{E}-02$ & $3.18 \mathrm{E}-02$ & $3.71 \mathrm{E}-02$ & $7.79 \mathrm{E}-02$ \\
8-hour Mission & $2.35 \mathrm{E}-02$ & $4.65 \mathrm{E}-02$ & $5.17 \mathrm{E}-02$ & $9.75 \mathrm{E}-02$ \\
\hline
\end{tabular}

a. By using industry-wide component failure data, individual plant performance is not included in the distribution of results. 


\section{INDUSTRY-WIDE TRENDS}

The yearly (1998-2018) failure and demand or run time data were obtained from ICES for the HPCI system. HPCI train maintenance unavailability data for trending are from the same time period, as reported in the ROP program and ICES. The component basic event uncertainty was calculated for the HPCI system components using the trending methods described in Section 1 and 2 of the Overview and Reference document [4]. Tables 6 and 7 show the yearly data values for each HPCI system specific component and failure mode combination that was varied in the model. These data were loaded into the HPCI system fault tree in each SPAR model with a HPCI system (see Table 1).

The trend charts show the results of varying component reliability data over time and updating generic, relatively-flat prior distributions using data for each year. In addition, for comparison, the calculated industry-wide system reliability this update (current SPAR/ ICES) is shown. Section 4 of the Overview and Reference document provides more detailed discussion of the trending methods. In the lower left hand corner of the trend figures, the regression method is reported.

The components that were varied in the HPCI model are:

- HPCI turbine-driven pump start, run, and test and maintenance.

- HPCI motor-operated valve, fail to operate.

Figure 1 shows the trend in the start-only model unreliability. Table 4 shows the data points for Figure 1. No statistically significant trend was identified within the industry-wide estimates of HPCI system unreliability (start-only) on a per fiscal year basis.

Figure 2 shows the trend in the 8-hour mission unreliability. Table 5 shows the data points for Figure 2. No statistically significant trend was identified within the industry-wide estimates of HPCI system unreliability (8-hour mission) on a per fiscal year basis. 


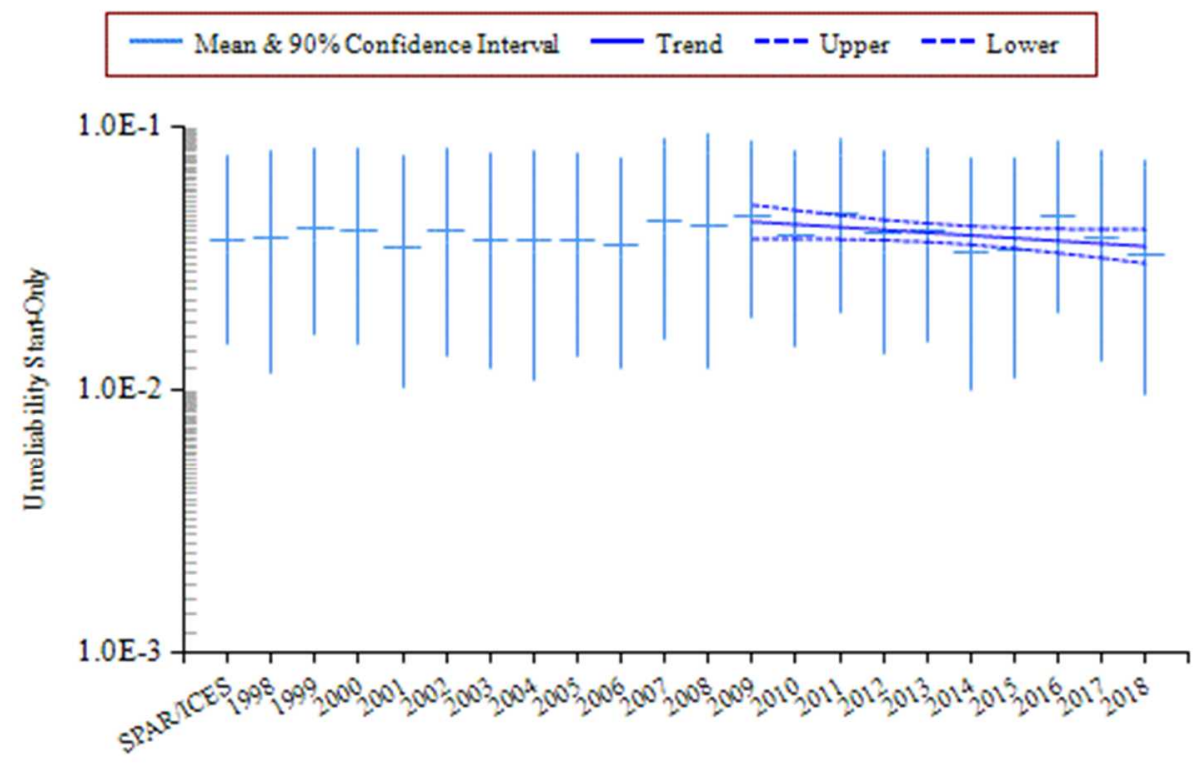

Calendar Year

LogisticTrend, $\mathrm{p}$-vahue $=$ 0.1020

HCI Industry Start-Only 12/2/2019

Figure 1. Trend of HPCI system unreliability (start-only model), as a function of fiscal year.

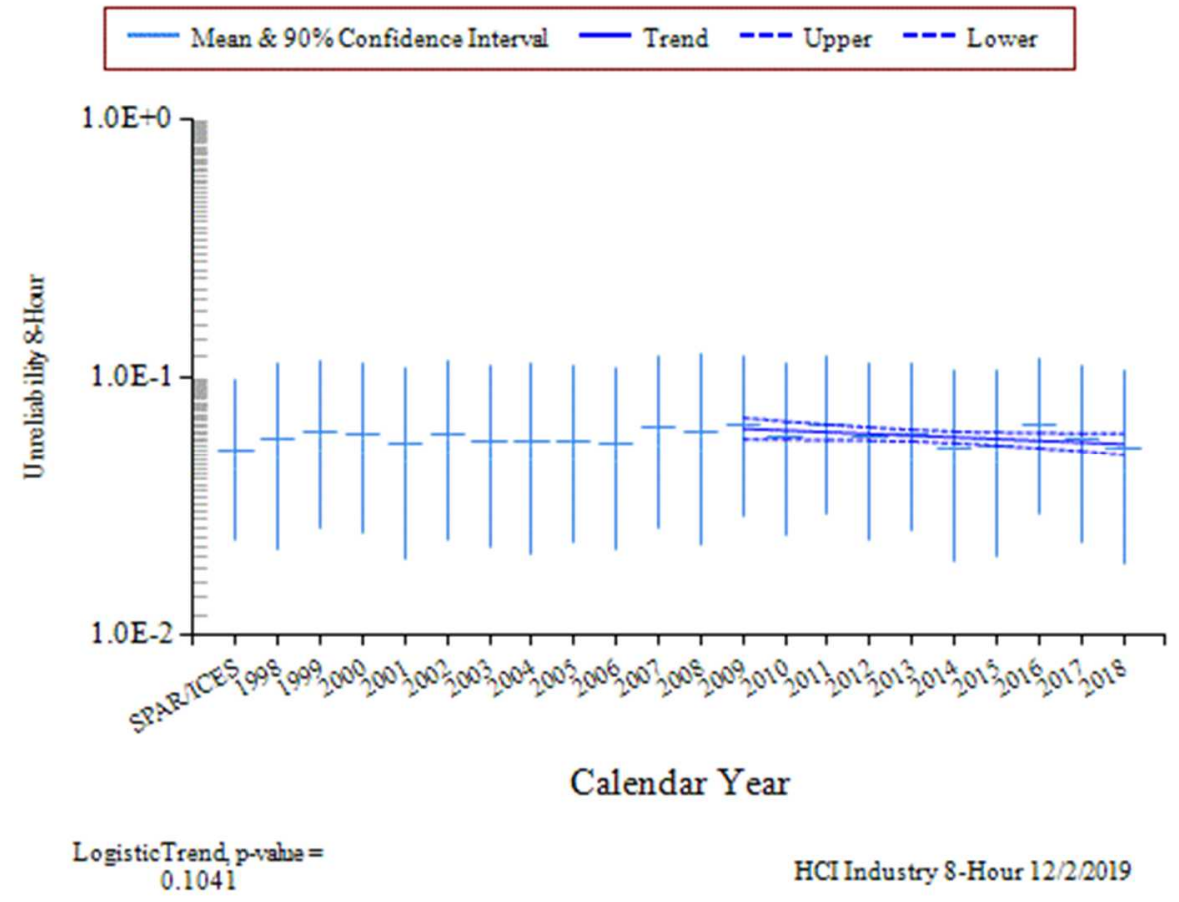

Figure 2. Trend of HPCI system unreliability (8-hour model), as a function of fiscal year. 


\section{BASIC EVENT GROUP IMPORTANCES}

The HPCI basic event group Fussell-Vesely importances were calculated for the start-only and 8-hour modes for each plant using the industry-wide data (1998-2010). These basic event group importances were then averaged across all plants to represent an industry-wide basic event group importance.

The industry-wide HPCI start-only and 8-hour basic event group importances are shown in Figure 3:

- In the Start-Only case - the leading contributor to HPCI system unreliability is the HPCI TDP group of basic events followed by the Injection group.

- In the 8-Hour case - the leading contributor to HPCI system unreliability is also the HPCI TDP group of basic events followed by the Injection group.

For more discussion on the HPCI turbine-driven pumps, see the turbine-driven pump component reliability study at the NRC Reactor Operational Experience Results and Databases web page (https://nrcoe.inl.gov/resultsdb/). Table 3 shows the SPAR model HPCI importance groups and their descriptions.

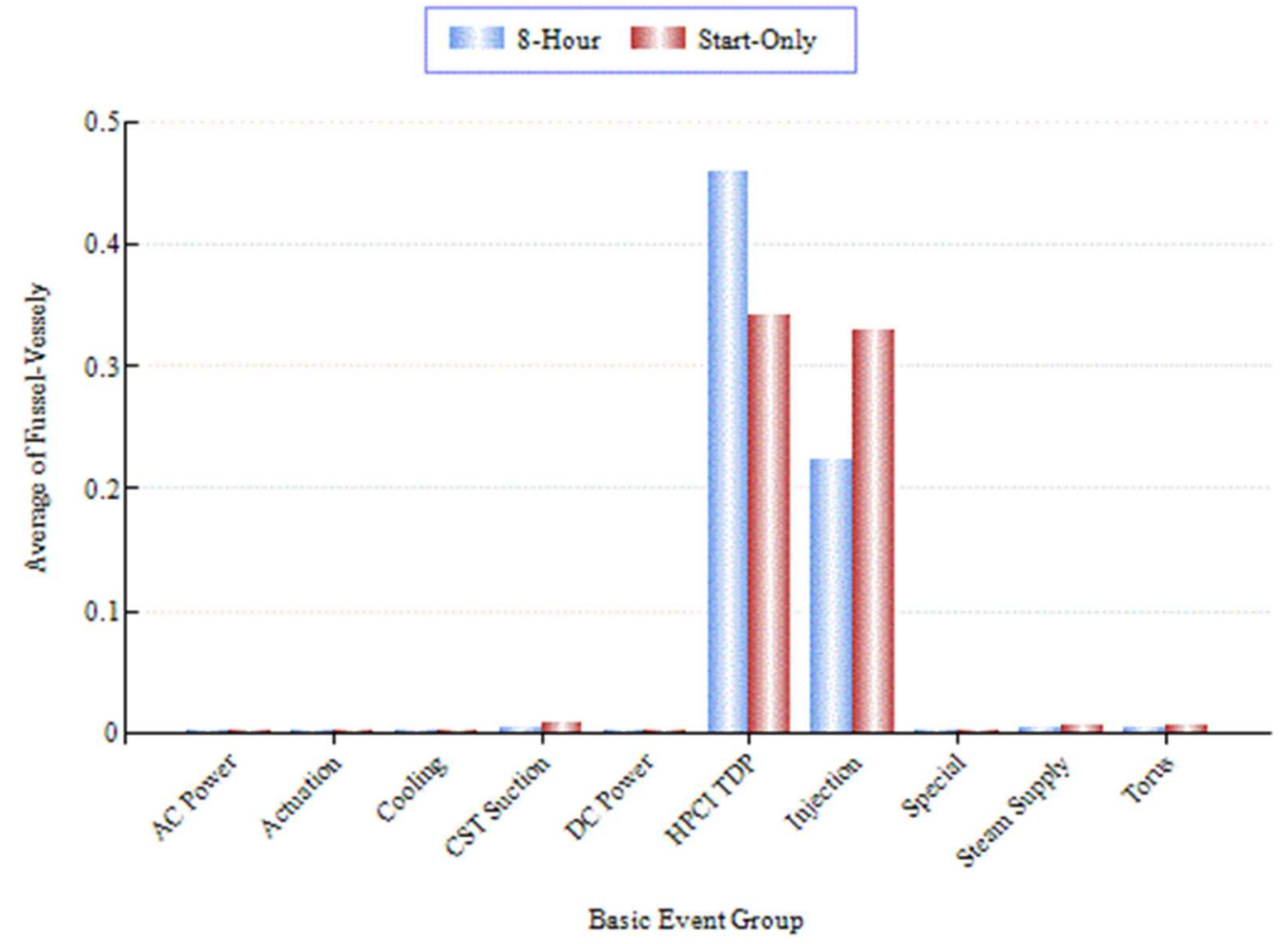

Figure 3. HPCI basic event group importances. 
Table 3. HPCI model basic event importance group descriptions.

\begin{tabular}{ll}
\hline \multicolumn{1}{c}{ Group } & \multicolumn{1}{c}{ Description } \\
\hline AC Power & $\begin{array}{l}\text { The ac buses and circuit breakers that supply power to the service water pumps } \\
\text { and the HPCI motor-operated valves. } \\
\text { ESF actuation circuitry. }\end{array}$ \\
$\begin{array}{l}\text { Actuation } \\
\text { Cooling }\end{array}$ & $\begin{array}{l}\text { The pumps, valves, and heat exchangers that provide heat removal to the HPCI } \\
\text { turbine-driven pump. }\end{array}$ \\
CST Suction & Suction path and condensate storage tank. \\
DC Power & $\begin{array}{l}\text { The batteries and battery chargers that supply power to the HPCI turbine-driven } \\
\text { pump control circuitry. }\end{array}$ \\
EPS & HPCl dependency on the emergency power system. \\
HPCI TDP & $\begin{array}{l}\text { All basic events associated with the turbine-driven pumps. The start, run, } \\
\text { common-cause, and test and maintenance are included in the group of basic } \\
\text { events. }\end{array}$ \\
Injection & The motor-operated valves and check valves in the HPCl injection path. \\
Steam Supply & The steam supply valves to the HPCl turbine. \\
Torus & $\begin{array}{l}\text { The suppression pool motor-operated valves, check valves, and strainers required } \\
\text { when a need to transfer to the suppression pool (torus) occurs. }\end{array}$ \\
\hline
\end{tabular}




\section{DATA TABLES}

Table 4. Plot data for HPCI start-only trend, Figure 1.

\begin{tabular}{|c|c|c|c|c|c|c|}
\hline \multirow[b]{2}{*}{ Year/Source } & \multicolumn{3}{|c|}{ Regression Curve Data Points } & \multicolumn{3}{|c|}{ Annual Estimate Data Points } \\
\hline & $\begin{array}{c}\text { Lower } \\
(5 \%)\end{array}$ & Mean & $\begin{array}{l}\text { Upper } \\
\text { (95\%) }\end{array}$ & $\begin{array}{c}\text { Lower } \\
(5 \%)\end{array}$ & Mean & $\begin{array}{l}\text { Upper } \\
(95 \%)\end{array}$ \\
\hline SPAR/ICES & -- & -- & -- & 1.49E-02 & 3.71E-02 & 7.79E-02 \\
\hline 1998 & -- & -- & -- & 1.16E-02 & 3.74E-02 & 8.04E-02 \\
\hline 1999 & -- & -- & -- & 1.63E-02 & 4.12E-02 & 8.29E-02 \\
\hline 2000 & -- & -- & -- & $1.50 \mathrm{E}-02$ & 4.04E-02 & 8.25E-02 \\
\hline 2001 & -- & -- & -- & 1.03E-02 & 3.50E-02 & 7.76E-02 \\
\hline 2002 & -- & -- & -- & 1.35E-02 & 3.98E-02 & 8.29E-02 \\
\hline 2003 & -- & -- & -- & 1.21E-02 & 3.70E-02 & 7.93E-02 \\
\hline 2004 & -- & -- & -- & 1.09E-02 & 3.69E-02 & 8.04E-02 \\
\hline 2005 & -- & -- & -- & 1.36E-02 & 3.70E-02 & 7.82E-02 \\
\hline 2006 & -- & -- & -- & 1.21E-02 & 3.51E-02 & 7.61E-02 \\
\hline 2007 & -- & -- & -- & 1.57E-02 & 4.40E-02 & 8.87E-02 \\
\hline 2008 & -- & -- & -- & 1.21E-02 & $4.21 \mathrm{E}-02$ & $9.41 \mathrm{E}-02$ \\
\hline 2009 & 3.75E-02 & 4.35E-02 & 5.04E-02 & 1.89E-02 & 4.59E-02 & 8.84E-02 \\
\hline 2010 & $3.75 \mathrm{E}-02$ & 4.25E-02 & 4.81E-02 & 1.46E-02 & 3.89E-02 & 8.02E-02 \\
\hline 2011 & 3.74E-02 & 4.15E-02 & 4.61E-02 & 1.94E-02 & 4.64E-02 & 8.87E-02 \\
\hline 2012 & 3.71E-02 & 4.05E-02 & 4.43E-02 & 1.36E-02 & 3.90E-02 & 8.11E-02 \\
\hline 2013 & 3.65E-02 & 3.96E-02 & 4.29E-02 & 1.53E-02 & 4.05E-02 & 8.23E-02 \\
\hline 2014 & 3.56E-02 & 3.86E-02 & 4.19E-02 & 1.01E-02 & 3.34E-02 & 7.50E-02 \\
\hline 2015 & $3.45 E-02$ & 3.77E-02 & 4.13E-02 & 1.10E-02 & 3.38E-02 & 7.49E-02 \\
\hline 2016 & 3.31E-02 & 3.68E-02 & 4.09E-02 & 1.95E-02 & 4.54E-02 & 8.70E-02 \\
\hline 2017 & 3.17E-02 & $3.60 \mathrm{E}-02$ & 4.08E-02 & 1.29E-02 & $3.80 \mathrm{E}-02$ & 8.02E-02 \\
\hline 2018 & 3.03E-02 & 3.51E-02 & 4.07E-02 & 9.58E-03 & 3.27E-02 & $7.42 \mathrm{E}-02$ \\
\hline
\end{tabular}


Table 5. Plot data for HPCI 8-hour trend, Figure 2.

\begin{tabular}{ccccccc}
\hline & \multicolumn{2}{c}{ Regression Curve Data Points } & \multicolumn{2}{c}{ Plot Trend Error Bar Points } \\
\cline { 2 - 7 } Year/Source & $\begin{array}{c}\text { Lower } \\
\mathbf{( 5 \% )}\end{array}$ & Mean & $\begin{array}{c}\text { Upper } \\
\mathbf{( 9 5 \% )}\end{array}$ & $\begin{array}{c}\text { Lower } \\
\mathbf{( 5 \% )}\end{array}$ & Mean & $\mathbf{1 9 5 \% )}$ \\
\hline SPAR/ICES & -- & -- & -- & $2.35 E-02$ & $5.17 E-02$ & $9.75 E-02$ \\
1998 & -- & -- & -- & $2.12 E-02$ & $5.75 E-02$ & $1.12 E-01$ \\
\hline 1999 & -- & -- & -- & $2.62 E-02$ & $6.12 E-02$ & $1.15 E-01$ \\
\hline 2000 & -- & -- & -- & $2.49 E-02$ & $6.04 E-02$ & $1.15 E-01$ \\
\hline 2001 & -- & -- & -- & $1.98 E-02$ & $5.51 E-02$ & $1.10 E-01$ \\
\hline 2002 & -- & -- & -- & $2.35 E-02$ & $5.98 E-02$ & $1.15 E-01$ \\
\hline 2003 & -- & -- & -- & $2.18 E-02$ & $5.71 E-02$ & $1.11 E-01$ \\
\hline 2004 & -- & -- & -- & $2.07 E-02$ & $5.69 E-02$ & $1.12 E-01$ \\
\hline 2005 & -- & -- & -- & $2.30 E-02$ & $5.71 E-02$ & $1.10 E-01$ \\
\hline 2006 & -- & -- & -- & $2.15 E-02$ & $5.53 E-02$ & $1.08 E-01$ \\
\hline 2007 & -- & -- & -- & $2.58 E-02$ & $6.39 E-02$ & $1.21 E-01$ \\
\hline 2008 & -- & -- & -- & $2.22 E-02$ & $6.20 E-02$ & $1.24 E-01$ \\
\hline 2009 & $5.77 E-02$ & $6.35 E-02$ & $6.98 E-02$ & $2.90 E-02$ & $6.58 E-02$ & $1.20 E-01$ \\
\hline 2010 & $5.76 E-02$ & $6.25 E-02$ & $6.78 E-02$ & $2.43 E-02$ & $5.89 E-02$ & $1.13 E-01$ \\
\hline 2011 & $5.75 E-02$ & $6.16 E-02$ & $6.59 E-02$ & $2.97 E-02$ & $6.63 E-02$ & $1.21 E-01$ \\
\hline 2012 & $5.72 E-02$ & $6.06 E-02$ & $6.42 E-02$ & $2.35 E-02$ & $5.91 E-02$ & $1.13 E-01$ \\
\hline 2013 & $5.67 E-02$ & $5.97 E-02$ & $6.29 E-02$ & $2.52 E-02$ & $6.05 E-02$ & $1.14 E-01$ \\
\hline 2014 & $5.58 E-02$ & $5.88 E-02$ & $6.20 E-02$ & $1.94 E-02$ & $5.36 E-02$ & $1.07 E-01$ \\
\hline 2015 & $5.46 E-02$ & $5.79 E-02$ & $6.14 E-02$ & $2.03 E-02$ & $5.40 E-02$ & $1.07 E-01$ \\
\hline 2016 & $5.32 E-02$ & $5.70 E-02$ & $6.11 E-02$ & $2.96 E-02$ & $6.53 E-02$ & $1.19 E-01$ \\
\hline 2017 & $5.18 E-02$ & $5.62 E-02$ & $6.09 E-02$ & $2.27 E-02$ & $5.81 E-02$ & $1.12 E-01$ \\
\hline 2018 & $5.02 E-02$ & $5.53 E-02$ & $6.09 E-02$ & $1.89 E-02$ & $5.29 E-02$ & $1.06 E-01$ \\
\hline
\end{tabular}


Table 6. Basic event reliability trending data.

\begin{tabular}{|c|c|c|c|c|c|c|c|c|}
\hline \multirow{2}{*}{$\begin{array}{c}\text { Failure } \\
\text { Mode }\end{array}$} & \multirow[b]{2}{*}{ Component } & \multirow[b]{2}{*}{ Year } & \multirow{2}{*}{$\begin{array}{l}\text { Number of } \\
\text { Failures }\end{array}$} & \multirow{2}{*}{$\begin{array}{l}\text { Demands/ } \\
\text { Run Hours }\end{array}$} & \multicolumn{4}{|c|}{ Bayesian Update } \\
\hline & & & & & Mean & Post A & Post B & Distribution \\
\hline FTOC & AOV & 1998 & 0 & 62 & 9.03E-04 & 1.11 & $1.23 E+03$ & Beta \\
\hline FTOC & AOV & 1999 & 0 & 62 & 9.03E-04 & 1.11 & $1.23 E+03$ & Beta \\
\hline FTOC & AOV & 2000 & 0 & 62 & 9.03E-04 & 1.11 & $1.23 E+03$ & Beta \\
\hline FTOC & AOV & 2001 & 0 & 62 & 9.03E-04 & 1.11 & $1.23 E+03$ & Beta \\
\hline FTOC & AOV & 2002 & 0 & 62 & 9.03E-04 & 1.11 & $1.23 E+03$ & Beta \\
\hline FTOC & AOV & 2003 & 0 & 62 & 9.03E-04 & 1.11 & $1.23 E+03$ & Beta \\
\hline FTOC & AOV & 2004 & 0 & 62 & 9.03E-04 & 1.11 & $1.23 E+03$ & Beta \\
\hline FTOC & AOV & 2005 & 0 & 62 & 9.03E-04 & 1.11 & $1.23 E+03$ & Beta \\
\hline FTOC & AOV & 2006 & 0 & 62 & 9.03E-04 & 1.11 & $1.23 E+03$ & Beta \\
\hline FTOC & AOV & 2007 & 0 & 62 & 9.03E-04 & 1.11 & $1.23 E+03$ & Beta \\
\hline FTOC & AOV & 2008 & 0 & 62 & 9.03E-04 & 1.11 & $1.23 E+03$ & Beta \\
\hline FTOC & AOV & 2009 & 0 & 62 & 9.03E-04 & 1.11 & $1.23 E+03$ & Beta \\
\hline FTOC & AOV & 2010 & 0 & 62 & 9.03E-04 & 1.11 & $1.23 E+03$ & Beta \\
\hline FTOC & AOV & 2011 & 0 & 62 & 9.03E-04 & 1.11 & $1.23 E+03$ & Beta \\
\hline FTOC & AOV & 2012 & 0 & 62 & 9.03E-04 & 1.11 & $1.23 E+03$ & Beta \\
\hline FTOC & AOV & 2013 & 0 & 62 & 9.03E-04 & 1.11 & $1.23 E+03$ & Beta \\
\hline FTOC & AOV & 2014 & 0 & 79 & 8.91E-04 & 1.11 & $1.25 \mathrm{E}+03$ & Beta \\
\hline FTOC & AOV & 2015 & 0 & 83 & 8.88E-04 & 1.11 & $1.25 \mathrm{E}+03$ & Beta \\
\hline FTOC & AOV & 2016 & 0 & 83 & 8.88E-04 & 1.11 & $1.25 \mathrm{E}+03$ & Beta \\
\hline FTOC & AOV & 2017 & 0 & 83 & 8.88E-04 & 1.11 & $1.25 \mathrm{E}+03$ & Beta \\
\hline FTOC & AOV & 2018 & 0 & 83 & 8.88E-04 & 1.11 & $1.25 \mathrm{E}+03$ & Beta \\
\hline FTOP & AOV & 1998 & 0 & 43,800 & 2.47E-07 & 1.42 & $5.76 \mathrm{E}+06$ & Gamma \\
\hline FTOP & AOV & 1999 & 0 & 43,800 & 2.47E-07 & 1.42 & $5.76 \mathrm{E}+06$ & Gamma \\
\hline FTOP & AOV & 2000 & 0 & 43,800 & 2.47E-07 & 1.42 & $5.76 \mathrm{E}+06$ & Gamma \\
\hline FTOP & AOV & 2001 & 0 & 43,800 & 2.47E-07 & 1.42 & $5.76 \mathrm{E}+06$ & Gamma \\
\hline FTOP & AOV & 2002 & 0 & 43,800 & 2.47E-07 & 1.42 & $5.76 \mathrm{E}+06$ & Gamma \\
\hline FTOP & AOV & 2003 & 0 & 43,800 & 2.47E-07 & 1.42 & $5.76 \mathrm{E}+06$ & Gamma \\
\hline FTOP & AOV & 2004 & 0 & 43,800 & 2.47E-07 & 1.42 & $5.76 \mathrm{E}+06$ & Gamma \\
\hline FTOP & AOV & 2005 & 0 & 43,800 & 2.47E-07 & 1.42 & $5.76 \mathrm{E}+06$ & Gamma \\
\hline FTOP & AOV & 2006 & 0 & 43,800 & 2.47E-07 & 1.42 & $5.76 \mathrm{E}+06$ & Gamma \\
\hline FTOP & AOV & 2007 & 0 & 43,800 & 2.47E-07 & 1.42 & $5.76 \mathrm{E}+06$ & Gamma \\
\hline FTOP & AOV & 2008 & 0 & 43,800 & 2.47E-07 & 1.42 & $5.76 \mathrm{E}+06$ & Gamma \\
\hline FTOP & AOV & 2009 & 0 & 43,800 & 2.47E-07 & 1.42 & $5.76 \mathrm{E}+06$ & Gamma \\
\hline FTOP & AOV & 2010 & 0 & 43,800 & 2.47E-07 & 1.42 & $5.76 \mathrm{E}+06$ & Gamma \\
\hline FTOP & AOV & 2011 & 0 & 43,800 & 2.47E-07 & 1.42 & $5.76 \mathrm{E}+06$ & Gamma \\
\hline FTOP & AOV & 2012 & 0 & 43,800 & 2.47E-07 & 1.42 & $5.76 \mathrm{E}+06$ & Gamma \\
\hline FTOP & AOV & 2013 & 0 & 43,800 & 2.47E-07 & 1.42 & $5.76 \mathrm{E}+06$ & Gamma \\
\hline FTOP & AOV & 2014 & 0 & 52,560 & $2.46 \mathrm{E}-07$ & 1.42 & $5.77 \mathrm{E}+06$ & Gamma \\
\hline FTOP & AOV & 2015 & 0 & 52,560 & 2.46E-07 & 1.42 & $5.77 \mathrm{E}+06$ & Gamma \\
\hline FTOP & AOV & 2016 & 0 & 52,560 & 2.46E-07 & 1.42 & $5.77 \mathrm{E}+06$ & Gamma \\
\hline FTOP & AOV & 2017 & 0 & 52,560 & 2.46E-07 & 1.42 & 5.77E+06 & Gamma \\
\hline
\end{tabular}


Table 6. (continued).

\begin{tabular}{|c|c|c|c|c|c|c|c|c|}
\hline \multirow{2}{*}{$\begin{array}{c}\text { Failure } \\
\text { Mode }\end{array}$} & \multirow[b]{2}{*}{ Component } & \multirow[b]{2}{*}{ Year } & \multirow{2}{*}{$\begin{array}{l}\text { Number of } \\
\text { Failures }\end{array}$} & \multirow{2}{*}{$\begin{array}{l}\text { Demands/ } \\
\text { Run Hours }\end{array}$} & \multicolumn{4}{|c|}{ Bayesian Update } \\
\hline & & & & & Mean & Post A & Post B & Distribution \\
\hline FTOP & AOV & 2018 & 0 & 52,560 & 2.46E-07 & 1.42 & $5.77 \mathrm{E}+06$ & Gamma \\
\hline SO & AOV & 1998 & 0 & 43,800 & 1.29E-07 & 0.68 & $5.25 \mathrm{E}+06$ & Gamma \\
\hline SO & AOV & 1999 & 0 & 43,800 & 1.29E-07 & 0.68 & $5.25 \mathrm{E}+06$ & Gamma \\
\hline SO & AOV & 2000 & 0 & 43,800 & $1.29 \mathrm{E}-07$ & 0.68 & $5.25 E+06$ & Gamma \\
\hline SO & AOV & 2001 & 0 & 43,800 & $1.29 \mathrm{E}-07$ & 0.68 & $5.25 \mathrm{E}+06$ & Gamma \\
\hline so & AOV & 2002 & 0 & 43,800 & 1.29E-07 & 0.68 & $5.25 \mathrm{E}+06$ & Gamma \\
\hline SO & AOV & 2003 & 0 & 43,800 & $1.29 \mathrm{E}-07$ & 0.68 & $5.25 \mathrm{E}+06$ & Gamma \\
\hline SO & AOV & 2004 & 0 & 43,800 & 1.29E-07 & 0.68 & $5.25 E+06$ & Gamma \\
\hline so & AOV & 2005 & 0 & 43,800 & 1.29E-07 & 0.68 & $5.25 \mathrm{E}+06$ & Gamma \\
\hline so & AOV & 2006 & 0 & 43,800 & $1.29 \mathrm{E}-07$ & 0.68 & $5.25 \mathrm{E}+06$ & Gamma \\
\hline so & AOV & 2007 & 0 & 43,800 & 1.29E-07 & 0.68 & $5.25 \mathrm{E}+06$ & Gamma \\
\hline SO & AOV & 2008 & 0 & 43,800 & 1.29E-07 & 0.68 & $5.25 \mathrm{E}+06$ & Gamma \\
\hline SO & AOV & 2009 & 0 & 43,800 & $1.29 \mathrm{E}-07$ & 0.68 & $5.25 \mathrm{E}+06$ & Gamma \\
\hline so & AOV & 2010 & 0 & 43,800 & $1.29 \mathrm{E}-07$ & 0.68 & $5.25 \mathrm{E}+06$ & Gamma \\
\hline so & AOV & 2011 & 0 & 43,800 & 1.29E-07 & 0.68 & $5.25 \mathrm{E}+06$ & Gamma \\
\hline SO & AOV & 2012 & 0 & 43,800 & 1.29E-07 & 0.68 & $5.25 \mathrm{E}+06$ & Gamma \\
\hline SO & AOV & 2013 & 0 & 43,800 & 1.29E-07 & 0.68 & $5.25 \mathrm{E}+06$ & Gamma \\
\hline SO & AOV & 2014 & 0 & 52,560 & 1.29E-07 & 0.68 & $5.26 \mathrm{E}+06$ & Gamma \\
\hline so & AOV & 2015 & 0 & 52,560 & 1.29E-07 & 0.68 & $5.26 \mathrm{E}+06$ & Gamma \\
\hline so & AOV & 2016 & 0 & 52,560 & 1.29E-07 & 0.68 & $5.26 \mathrm{E}+06$ & Gamma \\
\hline SO & AOV & 2017 & 0 & 52,560 & $1.29 \mathrm{E}-07$ & 0.68 & $5.26 \mathrm{E}+06$ & Gamma \\
\hline So & AOV & 2018 & 0 & 52,560 & 1.29E-07 & 0.68 & $5.26 \mathrm{E}+06$ & Gamma \\
\hline FTOC & MOV & 1998 & 5 & 2,110 & 1.66E-03 & 7.05 & $4.23 E+03$ & Beta \\
\hline FTOC & MOV & 1999 & 1 & 1,970 & 7.44E-04 & 3.05 & $4.09 \mathrm{E}+03$ & Beta \\
\hline FTOC & MOV & 2000 & 1 & 2,019 & 7.35E-04 & 3.05 & $4.14 \mathrm{E}+03$ & Beta \\
\hline FTOC & MOV & 2001 & 4 & 1,996 & 1.47E-03 & 6.05 & $4.11 \mathrm{E}+03$ & Beta \\
\hline FTOC & MOV & 2002 & 1 & 1,944 & 7.49E-04 & 3.05 & 4.07E+03 & Beta \\
\hline FTOC & MOV & 2003 & 1 & 2,097 & 7.21E-04 & 3.05 & $4.22 \mathrm{E}+03$ & Beta \\
\hline FTOC & MOV & 2004 & 2 & 1,984 & 9.85E-04 & 4.05 & $4.10 \mathrm{E}+03$ & Beta \\
\hline FTOC & MOV & 2005 & 4 & 1,989 & 1.47E-03 & 6.05 & $4.11 \mathrm{E}+03$ & Beta \\
\hline FTOC & MOV & 2006 & 1 & 1,996 & 7.39E-04 & 3.05 & $4.12 \mathrm{E}+03$ & Beta \\
\hline FTOC & MOV & 2007 & 8 & 2,036 & 2.41E-03 & 10.05 & $4.15 \mathrm{E}+03$ & Beta \\
\hline FTOC & MOV & 2008 & 4 & 2,033 & 1.45E-03 & 6.05 & $4.15 E+03$ & Beta \\
\hline FTOC & MOV & 2009 & 4 & 2,046 & 1.45E-03 & 6.05 & $4.16 \mathrm{E}+03$ & Beta \\
\hline FTOC & MOV & 2010 & 1 & 2,013 & 7.36E-04 & 3.05 & $4.13 E+03$ & Beta \\
\hline FTOC & MOV & 2011 & 0 & 1,958 & 5.01E-04 & 2.05 & $4.08 \mathrm{E}+03$ & Beta \\
\hline FTOC & MOV & 2012 & 3 & 1,984 & 1.23E-03 & 5.05 & $4.10 \mathrm{E}+03$ & Beta \\
\hline FTOC & MOV & 2013 & 1 & 1,984 & 7.41E-04 & 3.05 & $4.11 \mathrm{E}+03$ & Beta \\
\hline FTOC & MOV & 2014 & 1 & 1,954 & 7.47E-04 & 3.05 & $4.08 \mathrm{E}+03$ & Beta \\
\hline FTOC & MOV & 2015 & 1 & 1,934 & 7.51E-04 & 3.05 & $4.06 \mathrm{E}+03$ & Beta \\
\hline FTOC & MOV & 2016 & 1 & 1,965 & 7.45E-04 & 3.05 & $4.09 \mathrm{E}+03$ & Beta \\
\hline FTOC & MOV & 2017 & 1 & 1,969 & 7.44E-04 & 3.05 & $4.09 E+03$ & Beta \\
\hline
\end{tabular}


Table 6. (continued).

\begin{tabular}{|c|c|c|c|c|c|c|c|c|}
\hline \multirow{2}{*}{$\begin{array}{l}\text { Failure } \\
\text { Mode }\end{array}$} & \multirow[b]{2}{*}{ Component } & \multirow[b]{2}{*}{ Year } & \multirow{2}{*}{$\begin{array}{l}\text { Number of } \\
\text { Failures }\end{array}$} & \multirow{2}{*}{$\begin{array}{l}\text { Demands/ } \\
\text { Run Hours }\end{array}$} & \multicolumn{4}{|c|}{ Bayesian Update } \\
\hline & & & & & Mean & Post A & Post B & Distribution \\
\hline FTOC & MOV & 2018 & 0 & 1,836 & 5.16E-04 & 2.05 & $3.96 \mathrm{E}+03$ & Beta \\
\hline FTOP & MOV & 1998 & 0 & $2,137,440$ & 6.03E-08 & 1.46 & $2.42 \mathrm{E}+07$ & Gamma \\
\hline FTOP & MOV & 1999 & 0 & $2,128,680$ & 6.03E-08 & 1.46 & $2.42 \mathrm{E}+07$ & Gamma \\
\hline FTOP & MOV & 2000 & 0 & $2,128,680$ & $6.03 E-08$ & 1.46 & $2.42 \mathrm{E}+07$ & Gamma \\
\hline FTOP & MOV & 2001 & 0 & $2,128,680$ & 6.03E-08 & 1.46 & $2.42 \mathrm{E}+07$ & Gamma \\
\hline FTOP & MOV & 2002 & 0 & $2,128,680$ & $6.03 E-08$ & 1.46 & $2.42 \mathrm{E}+07$ & Gamma \\
\hline FTOP & MOV & 2003 & 0 & $2,128,680$ & 6.03E-08 & 1.46 & $2.42 \mathrm{E}+07$ & Gamma \\
\hline FTOP & MOV & 2004 & 0 & $2,137,440$ & $6.03 E-08$ & 1.46 & $2.42 \mathrm{E}+07$ & Gamma \\
\hline FTOP & MOV & 2005 & 0 & $2,137,440$ & 6.03E-08 & 1.46 & $2.42 \mathrm{E}+07$ & Gamma \\
\hline FTOP & MOV & 2006 & 0 & $2,137,440$ & $6.03 E-08$ & 1.46 & $2.42 \mathrm{E}+07$ & Gamma \\
\hline FTOP & MOV & 2007 & 1 & $2,137,440$ & 1.02E-07 & 2.46 & $2.42 \mathrm{E}+07$ & Gamma \\
\hline FTOP & MOV & 2008 & 0 & $2,137,440$ & 6.03E-08 & 1.46 & $2.42 \mathrm{E}+07$ & Gamma \\
\hline FTOP & MOV & 2009 & 0 & $2,154,960$ & 6.02E-08 & 1.46 & $2.42 \mathrm{E}+07$ & Gamma \\
\hline FTOP & MOV & 2010 & 0 & $2,207,520$ & $6.01 \mathrm{E}-08$ & 1.46 & $2.43 \mathrm{E}+07$ & Gamma \\
\hline FTOP & MOV & 2011 & 0 & $2,146,200$ & 6.03E-08 & 1.46 & $2.42 \mathrm{E}+07$ & Gamma \\
\hline FTOP & MOV & 2012 & 0 & $2,190,000$ & 6.01E-08 & 1.46 & $2.42 \mathrm{E}+07$ & Gamma \\
\hline FTOP & MOV & 2013 & 1 & $2,146,200$ & 1.02E-07 & 2.46 & $2.42 \mathrm{E}+07$ & Gamma \\
\hline FTOP & MOV & 2014 & 0 & $2,146,200$ & 6.03E-08 & 1.46 & $2.42 \mathrm{E}+07$ & Gamma \\
\hline FTOP & MOV & 2015 & 1 & $2,163,720$ & $1.02 \mathrm{E}-07$ & 2.46 & $2.42 \mathrm{E}+07$ & Gamma \\
\hline FTOP & MOV & 2016 & 0 & $2,111,160$ & 6.03E-08 & 1.46 & $2.42 \mathrm{E}+07$ & Gamma \\
\hline FTOP & MOV & 2017 & 0 & $2,111,160$ & 6.03E-08 & 1.46 & $2.42 \mathrm{E}+07$ & Gamma \\
\hline FTOP & MOV & 2018 & 0 & $2,111,160$ & 6.03E-08 & 1.46 & $2.42 \mathrm{E}+07$ & Gamma \\
\hline so & MOV & 1998 & 0 & $2,137,440$ & 3.01E-08 & 0.57 & $1.90 \mathrm{E}+07$ & Gamma \\
\hline SO & MOV & 1999 & 0 & $2,128,680$ & 3.01E-08 & 0.57 & $1.90 \mathrm{E}+07$ & Gamma \\
\hline SO & MOV & 2000 & 1 & $2,128,680$ & 8.28E-08 & 1.57 & $1.90 \mathrm{E}+07$ & Gamma \\
\hline SO & MOV & 2001 & 0 & $2,128,680$ & 3.01E-08 & 0.57 & $1.90 \mathrm{E}+07$ & Gamma \\
\hline SO & MOV & 2002 & 0 & $2,128,680$ & 3.01E-08 & 0.57 & $1.90 \mathrm{E}+07$ & Gamma \\
\hline SO & MOV & 2003 & 1 & $2,128,680$ & 8.28E-08 & 1.57 & $1.90 \mathrm{E}+07$ & Gamma \\
\hline SO & MOV & 2004 & 0 & $2,137,440$ & 3.01E-08 & 0.57 & $1.90 \mathrm{E}+07$ & Gamma \\
\hline SO & MOV & 2005 & 0 & $2,137,440$ & 3.01E-08 & 0.57 & $1.90 \mathrm{E}+07$ & Gamma \\
\hline so & MOV & 2006 & 0 & $2,137,440$ & 3.01E-08 & 0.57 & $1.90 \mathrm{E}+07$ & Gamma \\
\hline SO & MOV & 2007 & 1 & $2,137,440$ & 8.27E-08 & 1.57 & $1.90 \mathrm{E}+07$ & Gamma \\
\hline SO & MOV & 2008 & 0 & $2,137,440$ & 3.01E-08 & 0.57 & $1.90 \mathrm{E}+07$ & Gamma \\
\hline SO & MOV & 2009 & 0 & $2,154,960$ & $3.00 \mathrm{E}-08$ & 0.57 & $1.90 \mathrm{E}+07$ & Gamma \\
\hline SO & MOV & 2010 & 1 & $2,207,520$ & 8.24E-08 & 1.57 & $1.90 \mathrm{E}+07$ & Gamma \\
\hline SO & MOV & 2011 & 0 & $2,146,200$ & $3.00 \mathrm{E}-08$ & 0.57 & $1.90 \mathrm{E}+07$ & Gamma \\
\hline SO & MOV & 2012 & 0 & $2,190,000$ & 3.00E-08 & 0.57 & $1.90 \mathrm{E}+07$ & Gamma \\
\hline SO & MOV & 2013 & 0 & $2,146,200$ & $3.00 \mathrm{E}-08$ & 0.57 & $1.90 \mathrm{E}+07$ & Gamma \\
\hline SO & MOV & 2014 & 1 & $2,146,200$ & 8.27E-08 & 1.57 & $1.90 \mathrm{E}+07$ & Gamma \\
\hline SO & MOV & 2015 & 0 & $2,163,720$ & $3.00 \mathrm{E}-08$ & 0.57 & $1.90 \mathrm{E}+07$ & Gamma \\
\hline SO & MOV & 2016 & 0 & $2,111,160$ & 3.01E-08 & 0.57 & $1.90 \mathrm{E}+07$ & Gamma \\
\hline SO & MOV & 2017 & 0 & $2,111,160$ & 3.01E-08 & 0.57 & $1.90 \mathrm{E}+07$ & Gamma \\
\hline
\end{tabular}


Table 6. (continued).

\begin{tabular}{|c|c|c|c|c|c|c|c|c|}
\hline \multirow{2}{*}{$\begin{array}{l}\text { Failure } \\
\text { Mode }\end{array}$} & \multirow[b]{2}{*}{ Component } & \multirow[b]{2}{*}{ Year } & \multirow{2}{*}{$\begin{array}{l}\text { Number of } \\
\text { Failures }\end{array}$} & \multirow{2}{*}{$\begin{array}{l}\text { Demands/ } \\
\text { Run Hours }\end{array}$} & \multicolumn{4}{|c|}{ Bayesian Update } \\
\hline & & & & & Mean & Post A & Post B & Distribution \\
\hline SO & MOV & 2018 & 0 & $2,111,160$ & $3.01 \mathrm{E}-08$ & 0.57 & $1.90 \mathrm{E}+07$ & Gamma \\
\hline FTR $>1 \mathrm{H}$ & TDP & 1998 & 0 & 37 & $1.55 \mathrm{E}-03$ & 12.5 & $8.07 E+03$ & Gamma \\
\hline FTR $>1 \mathrm{H}$ & TDP & 1999 & 0 & 52 & $1.55 \mathrm{E}-03$ & 12.5 & $8.08 \mathrm{E}+03$ & Gamma \\
\hline FTR $>1 \mathrm{H}$ & TDP & 2000 & 1 & 35 & 1.67E-03 & 13.5 & $8.06 \mathrm{E}+03$ & Gamma \\
\hline $\mathrm{FTR}>1 \mathrm{H}$ & TDP & 2001 & 0 & 38 & $1.55 \mathrm{E}-03$ & 12.5 & $8.07 E+03$ & Gamma \\
\hline $\mathrm{FTR}>1 \mathrm{H}$ & TDP & 2002 & 0 & 23 & $1.55 \mathrm{E}-03$ & 12.5 & $8.05 E+03$ & Gamma \\
\hline $\mathrm{FTR}>1 \mathrm{H}$ & TDP & 2003 & 0 & 23 & 1.55E-03 & 12.5 & $8.05 E+03$ & Gamma \\
\hline $\mathrm{FTR}>1 \mathrm{H}$ & TDP & 2004 & 1 & 32 & 1.68E-03 & 13.5 & $8.06 E+03$ & Gamma \\
\hline $\mathrm{FTR}>1 \mathrm{H}$ & TDP & 2005 & 0 & 25 & $1.55 \mathrm{E}-03$ & 12.5 & $8.05 E+03$ & Gamma \\
\hline $\mathrm{FTR}>1 \mathrm{H}$ & TDP & 2006 & 0 & 18 & $1.55 \mathrm{E}-03$ & 12.5 & $8.05 E+03$ & Gamma \\
\hline FTR $>1 \mathrm{H}$ & TDP & 2007 & 0 & 18 & $1.55 \mathrm{E}-03$ & 12.5 & $8.05 E+03$ & Gamma \\
\hline $\mathrm{FTR}>1 \mathrm{H}$ & TDP & 2008 & 1 & 22 & $1.68 \mathrm{E}-03$ & 13.5 & $8.05 E+03$ & Gamma \\
\hline FTR $>1 \mathrm{H}$ & TDP & 2009 & 0 & 116 & 1.53E-03 & 12.5 & $8.14 \mathrm{E}+03$ & Gamma \\
\hline $\mathrm{FTR}>1 \mathrm{H}$ & TDP & 2010 & 1 & 57 & 1.67E-03 & 13.5 & $8.09 E+03$ & Gamma \\
\hline $\mathrm{FTR}>1 \mathrm{H}$ & TDP & 2011 & 2 & 28 & 1.80E-03 & 14.5 & $8.06 \mathrm{E}+03$ & Gamma \\
\hline $\mathrm{FTR}>1 \mathrm{H}$ & TDP & 2012 & 0 & 19 & $1.55 \mathrm{E}-03$ & 12.5 & $8.05 E+03$ & Gamma \\
\hline $\mathrm{FTR}>1 \mathrm{H}$ & TDP & 2013 & 1 & 33 & 1.67E-03 & 13.5 & $8.06 \mathrm{E}+03$ & Gamma \\
\hline $\mathrm{FTR}>1 \mathrm{H}$ & TDP & 2014 & 0 & 19 & $1.55 \mathrm{E}-03$ & 12.5 & $8.05 E+03$ & Gamma \\
\hline $\mathrm{FTR}>1 \mathrm{H}$ & TDP & 2015 & 0 & 22 & 1.55E-03 & 12.5 & $8.05 E+03$ & Gamma \\
\hline $\mathrm{FTR}>1 \mathrm{H}$ & TDP & 2016 & 1 & 30 & 1.68E-03 & 13.5 & $8.06 \mathrm{E}+03$ & Gamma \\
\hline $\mathrm{FTR}>1 \mathrm{H}$ & TDP & 2017 & 0 & 18 & $1.55 \mathrm{E}-03$ & 12.5 & $8.05 E+03$ & Gamma \\
\hline $\mathrm{FTR}>1 \mathrm{H}$ & TDP & 2018 & 0 & 18 & 1.55E-03 & 12.5 & $8.05 E+03$ & Gamma \\
\hline $\mathrm{FTR}<1 \mathrm{H}$ & TDP & 1998 & 0 & 219 & $2.21 \mathrm{E}-03$ & 0.96 & $4.35 \mathrm{E}+02$ & Gamma \\
\hline $\mathrm{FTR}<1 \mathrm{H}$ & TDP & 1999 & 0 & 211 & $2.25 \mathrm{E}-03$ & 0.96 & $4.28 \mathrm{E}+02$ & Gamma \\
\hline $\mathrm{FTR}<1 \mathrm{H}$ & TDP & 2000 & 1 & 201 & 4.70E-03 & 1.96 & $4.17 \mathrm{E}+02$ & Gamma \\
\hline $\mathrm{FTR}<1 \mathrm{H}$ & TDP & 2001 & 0 & 190 & 2.37E-03 & 0.96 & $4.06 \mathrm{E}+02$ & Gamma \\
\hline $\mathrm{FTR}<1 \mathrm{H}$ & TDP & 2002 & 0 & 185 & $2.40 \mathrm{E}-03$ & 0.96 & $4.02 \mathrm{E}+02$ & Gamma \\
\hline $\mathrm{FTR}<1 \mathrm{H}$ & TDP & 2003 & 2 & 192 & $7.25 \mathrm{E}-03$ & 2.96 & $4.09 E+02$ & Gamma \\
\hline $\mathrm{FTR}<1 \mathrm{H}$ & TDP & 2004 & 0 & 189 & $2.38 \mathrm{E}-03$ & 0.96 & $4.05 E+02$ & Gamma \\
\hline $\mathrm{FTR}<1 \mathrm{H}$ & TDP & 2005 & 0 & 194 & $2.35 \mathrm{E}-03$ & 0.96 & $4.10 \mathrm{E}+02$ & Gamma \\
\hline $\mathrm{FTR}<1 \mathrm{H}$ & TDP & 2006 & 0 & 205 & $2.28 \mathrm{E}-03$ & 0.96 & $4.21 \mathrm{E}+02$ & Gamma \\
\hline $\mathrm{FTR}<1 \mathrm{H}$ & TDP & 2007 & 3 & 197 & 9.58E-03 & 3.96 & $4.14 \mathrm{E}+02$ & Gamma \\
\hline $\mathrm{FTR}<1 \mathrm{H}$ & TDP & 2008 & 1 & 196 & 4.75E-03 & 1.96 & $4.13 E+02$ & Gamma \\
\hline $\mathrm{FTR}<1 \mathrm{H}$ & TDP & 2009 & 1 & 198 & 4.74E-03 & 1.96 & $4.14 \mathrm{E}+02$ & Gamma \\
\hline $\mathrm{FTR}<1 \mathrm{H}$ & TDP & 2010 & 0 & 195 & $2.34 \mathrm{E}-03$ & 0.96 & $4.11 \mathrm{E}+02$ & Gamma \\
\hline $\mathrm{FTR}<1 \mathrm{H}$ & TDP & 2011 & 0 & 196 & 2.33E-03 & 0.96 & $4.13 E+02$ & Gamma \\
\hline $\mathrm{FTR}<1 \mathrm{H}$ & TDP & 2012 & 0 & 190 & 2.36E-03 & 0.96 & $4.07 \mathrm{E}+02$ & Gamma \\
\hline $\mathrm{FTR}<1 \mathrm{H}$ & TDP & 2013 & 0 & 186 & $2.39 \mathrm{E}-03$ & 0.96 & $4.03 E+02$ & Gamma \\
\hline $\mathrm{FTR}<1 \mathrm{H}$ & TDP & 2014 & 0 & 175 & 2.46E-03 & 0.96 & $3.92 \mathrm{E}+02$ & Gamma \\
\hline $\mathrm{FTR}<1 \mathrm{H}$ & TDP & 2015 & 1 & 177 & 4.98E-03 & 1.96 & $3.94 \mathrm{E}+02$ & Gamma \\
\hline $\mathrm{FTR}<1 \mathrm{H}$ & TDP & 2016 & 2 & 193 & 7.23E-03 & 2.96 & $4.10 \mathrm{E}+02$ & Gamma \\
\hline $\mathrm{FTR}<1 \mathrm{H}$ & TDP & 2017 & 0 & 182 & $2.41 \mathrm{E}-03$ & 0.96 & $3.99 \mathrm{E}+02$ & Gamma \\
\hline
\end{tabular}


Table 6. (continued).

\begin{tabular}{|c|c|c|c|c|c|c|c|c|}
\hline \multirow{2}{*}{$\begin{array}{l}\text { Failure } \\
\text { Mode }\end{array}$} & \multirow[b]{2}{*}{ Component } & \multirow[b]{2}{*}{ Year } & \multirow{2}{*}{$\begin{array}{l}\text { Number of } \\
\text { Failures }\end{array}$} & \multirow{2}{*}{$\begin{array}{l}\text { Demands/ } \\
\text { Run Hours }\end{array}$} & \multicolumn{4}{|c|}{ Bayesian Update } \\
\hline & & & & & Mean & Post A & Post B & Distribution \\
\hline $\mathrm{FTR}<1 \mathrm{H}$ & TDP & 2018 & 0 & 172 & 2.47E-03 & 0.96 & $3.89 \mathrm{E}+02$ & Gamma \\
\hline FTS & TDP & 1998 & 0 & 219 & 2.59E-03 & 0.94 & $3.63 E+02$ & Beta \\
\hline FTS & TDP & 1999 & 3 & 211 & 1.11E-02 & 3.94 & $3.52 \mathrm{E}+02$ & Beta \\
\hline FTS & TDP & 2000 & 3 & 201 & 1.14E-02 & 3.94 & $3.42 E+02$ & Beta \\
\hline FTS & TDP & 2001 & 1 & 190 & 5.80E-03 & 1.94 & $3.33 E+02$ & Beta \\
\hline FTS & TDP & 2002 & 2 & 185 & 8.91E-03 & 2.94 & $3.27 \mathrm{E}+02$ & Beta \\
\hline FTS & TDP & 2003 & 1 & 192 & 5.76E-03 & 1.94 & $3.35 \mathrm{E}+02$ & Beta \\
\hline FTS & TDP & 2004 & 1 & 189 & 5.82E-03 & 1.94 & $3.32 E+02$ & Beta \\
\hline FTS & TDP & 2005 & 2 & 194 & 8.69E-03 & 2.94 & $3.36 \mathrm{E}+02$ & Beta \\
\hline FTS & TDP & 2006 & 2 & 205 & 8.41E-03 & 2.94 & $3.47 \mathrm{E}+02$ & Beta \\
\hline FTS & TDP & 2007 & 2 & 197 & 8.60E-03 & 2.94 & $3.39 \mathrm{E}+02$ & Beta \\
\hline FTS & TDP & 2008 & 2 & 196 & 8.62E-03 & 2.94 & $3.39 \mathrm{E}+02$ & Beta \\
\hline FTS & TDP & 2009 & 4 & 198 & 1.44E-02 & 4.94 & $3.38 \mathrm{E}+02$ & Beta \\
\hline FTS & TDP & 2010 & 2 & 195 & 8.66E-03 & 2.94 & 3.37E+02 & Beta \\
\hline FTS & TDP & 2011 & 4 & 196 & 1.45E-02 & 4.94 & $3.36 \mathrm{E}+02$ & Beta \\
\hline FTS & TDP & 2012 & 1 & 190 & 5.79E-03 & 1.94 & $3.33 E+02$ & Beta \\
\hline FTS & TDP & 2013 & 3 & 186 & 1.19E-02 & 3.94 & $3.27 \mathrm{E}+02$ & Beta \\
\hline FTS & TDP & 2014 & 0 & 175 & 2.94E-03 & 0.94 & $3.19 \mathrm{E}+02$ & Beta \\
\hline FTS & TDP & 2015 & 0 & 177 & 2.92E-03 & 0.94 & $3.21 \mathrm{E}+02$ & Beta \\
\hline FTS & TDP & 2016 & 4 & 193 & 1.46E-02 & 4.94 & $3.33 E+02$ & Beta \\
\hline FTS & TDP & 2017 & 1 & 182 & 5.93E-03 & 1.94 & $3.25 \mathrm{E}+02$ & Beta \\
\hline FTS & TDP & 2018 & 1 & 172 & $6.12 \mathrm{E}-03$ & 1.94 & $3.15 \mathrm{E}+02$ & Beta \\
\hline
\end{tabular}


Table 7. Basic event UA trending data.

\begin{tabular}{ccccccccc}
\hline \multirow{2}{*}{$\begin{array}{c}\text { Failure } \\
\text { Mode }\end{array}$} & Component & Year & UA & Critical & \multicolumn{3}{c}{ Bayesian Update } \\
\hline UA & TDP & 1998 & 2,417 & 182,188 & $1.42 \mathrm{E}-02$ & 1.72 & 119.62 & Beta \\
\hline UA & TDP & 1999 & 2,264 & 195,033 & $1.16 \mathrm{E}-02$ & 2.4 & 204.79 & Beta \\
\hline UA & TDP & 2000 & 2,047 & 196,240 & $1.04 \mathrm{E}-02$ & 1.56 & 148.15 & Beta \\
\hline UA & TDP & 2001 & 1,735 & 195,742 & $9.03 \mathrm{E}-03$ & 0.96 & 104.86 & Beta \\
\hline UA & TDP & 2002 & 2,403 & 199,989 & $1.21 \mathrm{E}-02$ & 1.39 & 113.05 & Beta \\
\hline UA & TDP & 2003 & 2,378 & 194,267 & $1.23 \mathrm{E}-02$ & 1.76 & 141.1 & Beta \\
\hline UA & TDP & 2004 & 2,240 & 196,465 & $1.16 \mathrm{E}-02$ & 1.12 & 95.13 & Beta \\
\hline UA & TDP & 2005 & 1,627 & 195,610 & $8.34 \mathrm{E}-03$ & 2.12 & 251.76 & Beta \\
\hline UA & TDP & 2006 & 1,535 & 197,941 & $7.81 \mathrm{E}-03$ & 1.91 & 243.38 & Beta \\
\hline UA & TDP & 2007 & 2,758 & 196,270 & $1.42 \mathrm{E}-02$ & 1.24 & 86.39 & Beta \\
\hline UA & TDP & 2008 & 2,718 & 199,399 & $1.36 \mathrm{E}-02$ & 0.58 & 42.2 & Beta \\
\hline UA & TDP & 2009 & 2,342 & 193,544 & $1.20 \mathrm{E}-02$ & 1.74 & 143.03 & Beta \\
\hline UA & TDP & 2010 & 2,263 & 197,083 & $1.14 \mathrm{E}-02$ & 2.5 & 216.3 & Beta \\
\hline UA & TDP & 2011 & 2,682 & 192,786 & $1.40 \mathrm{E}-02$ & 2.3 & 161.99 & Beta \\
\hline UA & TDP & 2012 & 2,632 & 192,525 & $1.35 \mathrm{E}-02$ & 2.04 & 148.48 & Beta \\
\hline UA & TDP & 2013 & 1,925 & 193,672 & $1.00 \mathrm{E}-02$ & 1.78 & 175.74 & Beta \\
\hline UA & TDP & 2014 & 2,198 & 196,708 & $1.12 \mathrm{E}-02$ & 2.12 & 186.94 & Beta \\
\hline UA & TDP & 2015 & 2,234 & 189,557 & $1.16 \mathrm{E}-02$ & 2.89 & 245.08 & Beta \\
\hline UA & TDP & 2016 & 2,416 & 192,415 & $1.25 \mathrm{E}-02$ & 2.84 & 225.2 & Beta \\
\hline UA & TDP & 2017 & 2,500 & 192,345 & $1.31 \mathrm{E}-02$ & 2.04 & 153.86 & Beta \\
\hline UA & TDP & 2018 & 1,475 & 188,839 & $7.86 \mathrm{E}-03$ & 1.36 & 171.46 & Beta \\
\hline
\end{tabular}

Table 8. Failure mode acronyms.

\begin{tabular}{ll}
\hline \multicolumn{1}{c}{ Failure Mode } & \multicolumn{1}{c}{ Failure Mode Description } \\
\hline FTOC & Fail to open/close \\
FTOP & Fail to operate \\
FTR $>1 \mathrm{H}$ & Fail to run greater than one hour \\
FTR $<1 \mathrm{H}$ & Fail to run less than one hour (after start) \\
FTS & Fail to start \\
SO & Spurious operation \\
UA & Unavailability (maintenance or state of another component) \\
\hline
\end{tabular}




\section{SYSTEM DESCRIPTION}

The HPCI system is a single-train system that provides a reliable source of high-pressure coolant for cases where there is a loss of normal core coolant inventory. Figure 4 provides a simplified schematic diagram of the system.

The HPCI system consists of a steam turbine-driven pump, valves, and valve operators, and associated piping, including that from the normal and alternate pump suction sources and the pump discharge up to the penetration of the main feedwater line. For this study, the part of the main feedwater line from the check valve upstream of the HPCI connection to the reactor vessel, including the check valve, was considered part of the HPCI system. The steam turbine-driven pump includes all steam piping from the main steam line penetration to the turbine, and turbine exhaust piping to the suppression pool, valves and valve operators, gland sealing steam, and the turbine auxiliary oil system.

The HPCI system is actuated by either a low reactor water level or a high drywell pressure. Initially the system operates in an open loop mode, taking suction from the condensate storage tank (CST) and injecting water into the reactor pressure vessel (RPV) via one of the main feedwater lines. When the level in the CST reaches a low-level set point, the HPCI pump suction is aligned to the suppression pool. To maintain RPV level after the initial recovery, the HPCI system is placed in manual control, which may involve controlling turbine speed, diverting flow through minimum-flow or test lines, cycling the injection motor-operated valve (MOV), or complete stop-start cycles.

The HPCI system is also manually used to help control RPV pressure following a transient. Although this is not part of the ECCS design function, it is employed in this manner in PRAs. However, only a small percentage PRAs that employ this function model the pressure control operation. In this mode, the turbine-driven pump is operated manually with the injection valve closed and the full-flow test-line MOV open. Turbine operation with the injection line isolated and the test line open allows the turbine to draw steam from the RPV, thereby reducing RPV pressure. Operation of the system in the pressure control mode may also occur with intermittent injection of coolant to the RPV. As steam is being drawn off the RPV, the RPV water inventory is reduced, resulting in the need for vessel water level restoration. When level restoration is required, the injection valve is opened and the test-line MOV is closed. Upon restoration of RPV water inventory, the system is returned to the pressure control line-up. This cycling between injection and pressure control can be repeated as necessary. 


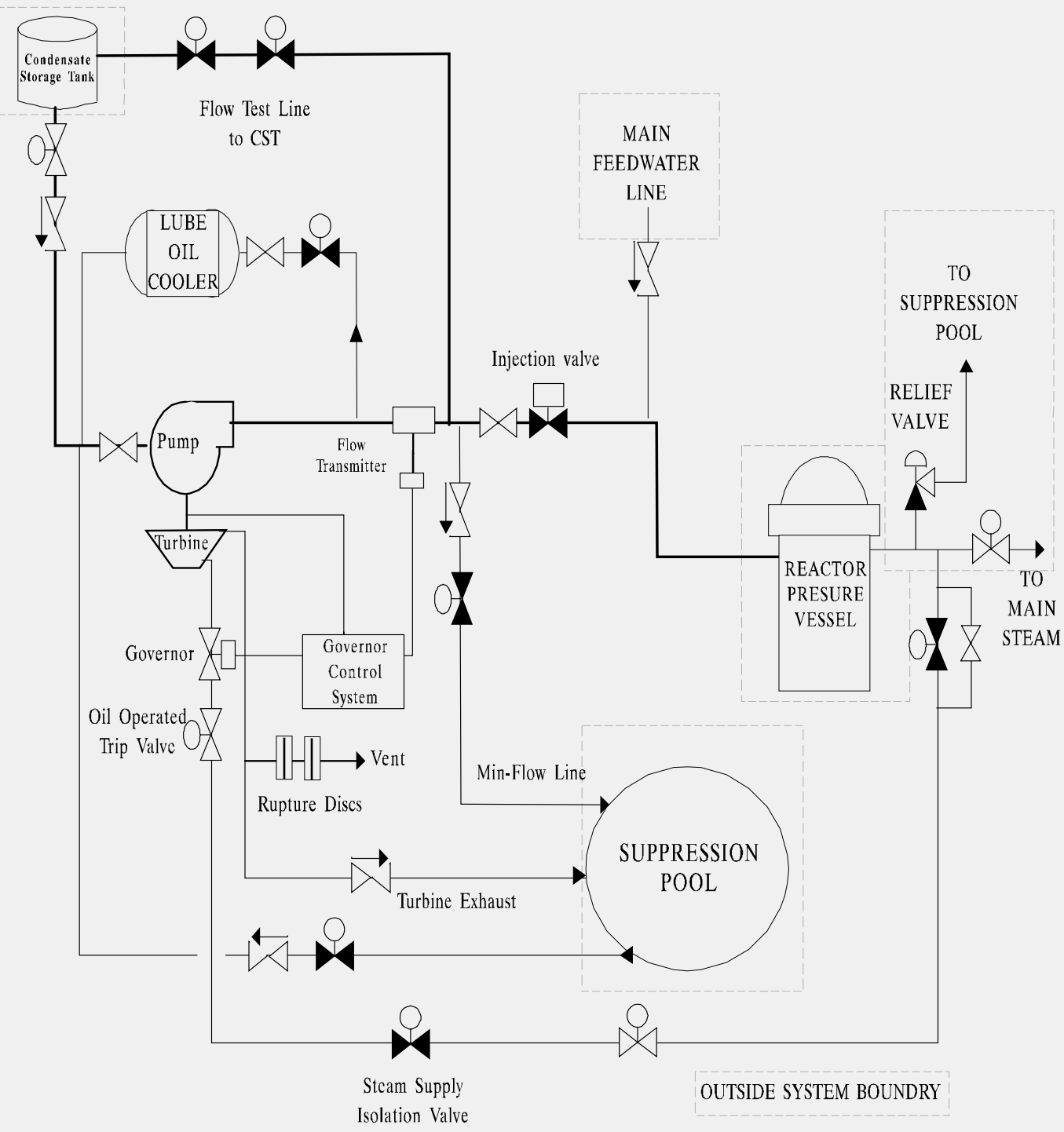

BWR HIGH PRESSURE COOLANT INJECTION SYSTEM

Figure 4. HPCI system diagram. 


\section{REFERENCES}

[1] United States Nuclear Regulatory Commission, "Component Reliability Data Sheets Update 2015," February 2017. [Online]. Available:

https://nrcoe.inl.gov/resultsdb/publicdocs/AvgPerf/ComponentUR2015.pdf.

[2] S. A. Eide, T. E. Wierman, C. D. Gentillon, D. M. Rasmuson and C. L. Atwood, "Industry-Average Performance for Components and Initiating Events at U.S. Commercial Nuclear Power Plants,"

NUREG/CR-6928, U.S. Nuclear Regulatory Commission, February 2007.

[3] United States Nuclear Regulatory Commission, "Component Reliability Data Sheets Update 2010," January 2012. [Online]. Available:

https://nrcoe.inl.gov/resultsdb/publicdocs/AvgPerf/ComponentUR2010.pdf.

[4] C. D. Gentillion, "Overview and Reference Document for Operational Experience Results and Databases Trending," February 2016. [Online]. Available:

https://nrcoe.inl.gov/resultsdb/publicdocs/Overview-and-Reference.pdf. 